\title{
DEGRADAÇÃO E ESTABILIZAÇÃO DO DICLOFENACO EM NANOCÁPSULAS POLIMÉRICAS
}

\author{
Claudia Regina Müller, Sandra Elisa Haas, Valquíria Linck Bassani e Sílvia Stanisçuaski Guterres*
}

Faculdade de Farmácia, Universidade Federal do Rio Grande do Sul, Av. Ipiranga, 2752, 90610-000 Porto Alegre - RS

Hatem Fessi

Laboratoire d’Automatique et de Génie des Procédés, Université de Lyon I, França

Maria do Carmo R. Peralba e Adriana Raffin Pohlmann

Instituto de Química, Universidade Federal do Rio Grande do Sul, CP 15003, 91501-970 Porto Alegre - RS

Recebido em 2/6/03; aceito em 18/2/04; publicado na web em 17/06/04

\begin{abstract}
DEGRADATION AND STABILIZATION OF DICLOFENAC IN POLYMERIC NANOCAPSULES. The parameters which affect the degradation and stabilization of diclofenac in suspensions of nanocapsules and of the corresponding spray-dried powders were investigated. Formulations were subjected to 14 months of storage at room temperature. In addition, a study of the degradation of diclofenac was carried out by exposing the formulations or mixtures (drug and adjuvants) to UVC wavelengths. The presence of Epikuron $170^{\circledR}$ in a concentration higher than $3.06 \mathrm{mg} / \mathrm{mL}$ stabilizes the drug, avoiding its reduction or degradation. The degradation products were isolated, analyzed by gas chromatography-mass spectrometry, and identified as $2-\left(2^{\prime}, 6^{\prime}-\right.$ dichlorophenyl)aminobenzyl alcohol and $N$-(2',6'-dichlorophenyl)anthranilylaldehyde.
\end{abstract}

Keywords: nanoparticles; diclofenac; degradation.

\section{INTRODUÇÃo}

Embora os antiinflamatórios não-esteróides (AINE) constituam uma das classes de fármacos mais prescritas no mundo, seu uso como antiinflamatório continua sendo limitado pela incidência de seus efeitos indesejados, principalmente sobre o trato gastrintestinal, que incluem irritação, sangramento, ulceração e, eventualmente, perfuração da parede gastrintestinal ${ }^{1,2}$. Existem vários estudos que mostram a relação entre o tratamento com diclofenaco e o aparecimento de lesões gastrintestinais, tanto no homem como em animais ${ }^{3-7}$. Diversos mecanismos são propostos para explicar a toxicidade digestiva dos AINE. As lesões gastrintestinais produzidas em animais de laboratórios, pela administração de AINE, são conseqüência de um mecanismo de ação local, pelo contato direto do fármaco em grandes concentrações com a mucosa gastrintestinal, e de um mecanismo de ação sistêmica, que aparece após a absorção ${ }^{7-9}$.

A toxicidade gastrintestinal do diclofenaco, um AINE, motivou o desenvolvimento de várias formas farmacêuticas e sistemas dispersos com o objetivo de reduzir esses efeitos colaterais, tais como sua formulação em micelas ${ }^{10}$, lipossomas ${ }^{11}$, associação com prostaglandinas $^{12}$, associação com ciclodextrinas ${ }^{13}$, microcápsulas ${ }^{14,15}$ e, mais recentemente, nanocápsulas ${ }^{16}$ e nanoesferas ${ }^{17}$. Nanocápsulas de ácido poli(D,L-lático) contendo diclofenaco na forma ácida foram preparadas por Guterres e colaboradores ${ }^{16}$. A avaliação da toxicidade gastrintestinal destas formas, em ratos, demonstrou uma significativa proteção da mucosa gastrintestinal após a administração do fármaco nanoencapsulado, quando comparada com a administração de uma solução aquosa deste fármaco. Os mesmos resultados foram observados por Müller ${ }^{17}$, quando foram avaliadas suspensões de nanoesferas de poli( $\varepsilon$-caprolactona) contendo diclofenaco na forma ácida. Guterres e colaboradores ${ }^{18}$ também avaliaram a toxicidade gastrintestinal de formas sólidas obtidas a partir das suspensões de nanocápsulas e nanoesferas de poli( $\varepsilon$-caprolactona), con-

*e-mail: nanoc@farmacia.ufrgs.br tendo diclofenaco, secas por aspersão ${ }^{19}$. O estudo demonstrou que tanto os produtos de nanocápsulas quanto os de nanoesferas, secos por aspersão, apresentaram um efeito protetor da mucosa gastrintestinal. Schaffazick ${ }^{20}$ avaliou a toxicidade gastrintestinal de nanopartículas liofilizadas contendo diclofenaco. Os liofilizados de nanoesferas ou nanocápsulas apresentaram um efeito protetor sobre a mucosa gastrintestinal similar àquele observado nos estudos realizados por Müller ${ }^{17}$ e Guterres e colaboradores ${ }^{18}$, sobre a associação de diclofenaco com nanopartículas poliméricas.

Apesar dos excelentes resultados observados nos experimentos de tolerância digestiva do diclofenaco quando associado a nanocápsulas ${ }^{18}$, estudos de estabilidade de suspensões coloidais, contendo os tensoativos monoestearato de sorbitano e Tween $80^{\circledR}$ e poli $(\varepsilon$ caprolactona) como polímero, demonstraram que o teor de diclofenaco diminuía em função do tempo de armazenamento ${ }^{17}$. Nestes estudos, utilizando cromatografia líquida de alta eficiência (CLAE), foi observada uma diminuição significativa na quantidade do diclofenaco após 3 meses de fabricação e o aparecimento de dois picos, prováveis produtos de degradação do fármaco. A área destes picos aumentava com o tempo, na medida em que diminuía a área do pico referente ao diclofenaco.

Diversos relatos na literatura têm apontado a degradação do diclofenaco, tanto em solução aquosa como no estado sólido, decorrentes de uma ciclização indolinônica, de fotodegradação, de degradação térmica ou de oxidação ${ }^{21-29}$. Os produtos de degradação do diclofenaco, já relatados, são os resultantes de ciclização indolinônica, formando o 1-(2',6'-diclorofenil)oxoindol ${ }^{21,26}$ e o 2,6-diclorofenol ${ }^{22,23}$, de fotodegradação, formando o ácido 8-clorocarbazol-1-acético e o ácido carbazol-1-acético ${ }^{24,29}$, de esterificação, formando o éster metílico do diclofenaco ${ }^{26}$, de oxidação, formando a 1-(2',6'diclorofenil)isatina ${ }^{26}$ e de degradação térmica, formando o 4-cloro$(10 H)$-9-acridinona ${ }^{25}$. Além destes, outros dois produtos de degradação também foram identificados, o álcool 2-(2',6'-diclorofenil)aminobenzilíco e $N$-(2',6'-diclorofenil)antranilaldeído ${ }^{30}$. Por outro lado, não há relatos na literatura de estudos de degradação desse fármaco, quando associado a nanopartículas poliméricas. 
Os produtos de degradação do diclofenaco, anteriormente detectados através de CLAE, após 3 meses de armazenamento das suspensões de nanocápsulas preparadas por Müller ${ }^{17}$, não foram até o presente momento identificados. Neste contexto, o presente trabalho referese a um estudo da degradação do diclofenaco a partir das suspensões de nanocápsulas e de seus produtos secos por aspersão, verificando os possíveis fatores que conduzem à degradação do fármaco em suspensões coloidais e identificando os produtos de degradação formados. A avaliação da influência da composição do sistema de tensoativos, empregados para a formulação dos produtos, também é foco do trabalho, com vista ao aumento da estabilidade dos sistemas.

\section{PARTE EXPERIMENTAL}

\section{Materiais}

Todos os materiais utilizados na fabricação das suspensões de nanocápsulas e dos produtos secos por aspersão apresentaram grau farmacêutico, sendo utilizados como recebidos: diclofenaco de sódio (Sigma, St. Louis - MO), poli(E-caprolactona) PM 45 a $60 \mathrm{kDa}$ (Aldrich, Strasburg, França), Epikuron $170^{\circledR}$ (mistura de fosfo- e glico-lipídeos de soja) (Lucas Meyer, Hambourg, Alemanha), Tween $80^{\circledR}$ (polissorbato 80) (Distribuidora Delaware, Porto Alegre, Brasil), Miglyol $810^{\circledR}$ - mistura de triglicerídeos dos ácidos cáprico e caprílico (Hulls, Puteaux, France) e dióxido de silício coloidal (Aerosil 200 ${ }^{\circledR}$, Degussa, São Paulo, Brasil).

\section{Suspensões de nanocápsulas}

O diclofenaco sódico foi previamente transformado na sua forma ácida ${ }^{31}$, para possibilitar sua associação às nanocápsulas. As suspensões coloidais foram preparadas pelo método de deposição interfacial de um polímero pré-formado (nanoprecipitação), proposto por Fessi e colaboradores $^{32}$. Diferentes formulações foram preparadas, conforme o seguinte procedimento: em um frasco, foram dissolvidos, em acetona $(267 \mathrm{~mL})$, o diclofenaco ácido $(0,10 \mathrm{~g})$, a poli( $(\varepsilon$-caprolactona) $(1,00 \mathrm{~g})$, o Miglyol $810^{\circledR}(3,30 \mathrm{~g})$ e o Epikuron $170^{\circledR}$ em concentrações crescentes para as diferentes suspensões (Tabela 1), sob agitação à temperatura de $48{ }^{\circ} \mathrm{C}$. Separadamente, o Tween $80^{\circledR}$ (Tabela 1) foi dissolvido em água destilada $(533 \mathrm{~mL})$ à temperatura ambiente. Em seguida, as diferentes fases orgânicas, totalmente solubilizadas, foram vertidas lentamente e sob agitação moderada, à temperatura ambiente, através de um funil, sobre cada fase aquosa. As suspensões foram mantidas sob agitação moderada durante 10 min à temperatura ambiente. A seguir, procedeu-se à evaporação sob pressão reduzida de cada suspensão de nanocápsulas em evaporador rotatório, a um volume final de $100 \mathrm{~mL}$ para eliminação da acetona e ajuste da concentração final do diclofenaco $(1 \mathrm{mg} / \mathrm{mL})$. Em seguida, as suspensões foram armazenadas em frascos de vidro âmbar.

A medida da quantidade total de diclofenaco em cada suspensão foi realizada através da dissolução das nanoestruturas pelo tratamen-

Tabela 1. Quantidades de tensoativos utilizadas nas formulações de nanocápsulas

\begin{tabular}{ccc}
\hline Suspensão & Epikuron $170^{\circledR}(\mathrm{mg})$ & Tween $80^{\circledR}(\mathrm{mg})$ \\
\hline A & 766 & 766 \\
B & 613 & 613 \\
C & 306 & 306 \\
D & 153 & 153 \\
E & 766 & -766 \\
F & - & \\
G & - & \\
\hline
\end{tabular}

to das mesmas com acetonitrila. Utilizou-se a CLAE (Cromatógrafo Perkin Elmer, Series 200), com detecção em 280 nm, para sua quantificação, utilizando metodologia previamente validada por Müller ${ }^{17}$. Empregou-se pré-coluna com fase estacionária LiChrosorb RP18 - 3,9 mm $\times 50 \mathrm{~mm}$ (Merck) acoplada à coluna Nova-Pak ${ }^{\circledR}$ C18 - 3,9 × $150 \mathrm{~mm}$ (Waters). A fase móvel foi constituída de mistura de acetonitrila/água $(65: 35 \mathrm{v} / \mathrm{v})$, ajustada a $\mathrm{pH}$ aparente 5,0 com ácido acético $10 \%$, fluxo de $0,7 \mathrm{~mL} / \mathrm{min}$ e volume injetado de amostra de $20 \mu \mathrm{L}$. O doseamento do diclofenaco foi realizado logo após a preparação das suspensões e após 3, 6 e 14 meses de armazenamento à temperatura ambiente.

Após a preparação, o diâmetro médio das nanocápsulas foi determinado por espectroscopia de auto-correlação da difusão de raio monocromático laser (Coulter ${ }^{\circledR}$ Granulomètre Laser LS 230, Small Volume Module, Coulter Corporation, Miami, Flórida). Os resultados representam a média das determinações de 3 lotes de formulações.

\section{Produtos secos por aspersão}

Os pós foram obtidos a partir da secagem em torre Mini Spraydryer Büchi $190^{\circledR}$ por aspersão ${ }^{19,33}$ das diferentes suspensões de nanocápsulas (Tabela 1) contendo diclofenaco, utilizando-se 3\% (m/v) de Aerosil $200^{\circledR}$ como adjuvante de secagem. A temperatura do ar de entrada foi de $150{ }^{\circ} \mathrm{C} \pm 4{ }^{\circ} \mathrm{C}$ e a de saída de $110^{\circ} \mathrm{C} \pm 4{ }^{\circ} \mathrm{C}$, utilizando-se um fluxo de alimentação de $3 \mathrm{~mL} / \mathrm{min}$.

O diclofenaco foi quantificado logo após a obtenção dos pós e após 3, 6 e 14 meses de armazenamento à temperatura ambiente, adicionando-se, a uma determinada quantidade do produto seco, $30 \mathrm{~mL}$ de acetonitrila em balão volumétrico de $50 \mathrm{~mL}$. Cada mistura foi submetida à agitação em agitador magnético, durante $60 \mathrm{~min}$, para a extração do diclofenaco. Posteriormente o volume foi completado a $50 \mathrm{~mL}$. Em seguida, as amostras foram filtradas através de membrana hidrofílica (GVWP, 0,22 $\mu$ m, Millipore), com auxílio de seringa, transferidas para frascos de injeção automática e submetidas à análise por CLAE, conforme metodologia descrita para a quantificação do fármaco nas suspensões.

\section{Experimentos de degradação}

O estudo de fotodegradação foi realizado em uma câmara de reação composta por uma câmara de $100 \mathrm{~cm}$ de comprimento, $16 \mathrm{~cm}$ de altura e $16 \mathrm{~cm}$ de profundidade, espelhada internamente, equipada com lâmpada germicida "Phillips TUV lamp - UVC long life", de $30 \mathrm{~W}$ de potência e $96 \mathrm{~V}$ de voltagem ${ }^{34}$. A temperatura interna foi mantida em $28^{\circ} \mathrm{C} \pm 1{ }^{\circ} \mathrm{C}$.

Foram submetidas à fotodegradação, amostras de diclofenaco na forma ácida, uma suspensão de nanocápsulas poliméricas contendo diclofenaco e seu produto seco por aspersão correspondente (Tabela 1, formulação A), uma suspensão de nanocápsulas poliméricas contendo diclofenaco formulada sem o Epikuron $170^{\circledR}$ e seu produto correspondente seco por aspersão (Tabela 1). Também foram submetidos à fotodegradação, todos os componentes de uma formulação de nanocápsulas (Miglyol $810^{\circledR}$, Tween $80^{\circledR}$, poli( $(\varepsilon$-caprolactona) e Epikuron $170^{\circledR}$ ) e misturas binárias dos mesmos com o diclofenaco ácido.

Para verificar a possível influência da temperatura interna da câmara de reação sobre a degradação do fármaco, uma suspensão de nanocápsulas poliméricas contendo diclofenaco formulada sem o Epikuron $170^{\circledR}$ e seu produto seco correspondente (Tabela 1, formulação F) também foram ensaiados em frascos cobertos com papel alumínio.

As amostras sólidas foram colocadas em vidro de relógio e as amostras líquidas, em Becker. Foram realizadas coletas das amostras 
após 1, 2, 3 e 14 dias de exposição à luz UVC. Todas as amostras coletadas foram solubilizadas em acetonitrila e analisadas qualitativamente por CLAE, de acordo com metodologia descrita acima para a detecção do diclofenaco nas suspensões.

\section{Isolamento dos produtos de degradação do diclofenaco}

A extração e o isolamento dos produtos de degradação do diclofenaco foram realizados a partir de um produto de nanocápsulas, seco por aspersão, contendo diclofenaco, armazenado durante dois anos, e que apresentava degradação do fármaco, confirmada através de análise por CLAE. A composição desta formulação consistia de diclofenaco ácido, poli( $\varepsilon$-caprolactona), Miglyol 810 ${ }^{\circledR}$, monoestearato de sorbitano, Tween $80^{\circledR}$ e Aerosil $200^{\circledR 17}$.

Para extração do diclofenaco e/ou produtos de degradação, um grama deste pó foi disperso em $50 \mathrm{~mL}$ de acetonitrila e submetido à agitação em agitador magnético durante $1 \mathrm{~h}$, na temperatura ambiente. Em seguida, esta dispersão foi submetida à filtração, sob vácuo, em funil de vidro sinterizado G3, contendo $5 \mathrm{~g}$ de gel de sílica para coluna (230-400 mesh) para retirada do adjuvante de secagem (Aerosil $\left.200^{\circledast}\right)$. O filtrado foi submetido à evaporação até secura, em evaporador rotatório, obtendo-se um resíduo.

Numa segunda etapa, o objetivo foi separar o polímero dos demais componentes da formulação no resíduo obtido na etapa anterior. Diretamente no balão de fundo redondo contendo o resíduo da evaporação, adicionou-se $50 \mathrm{~mL}$ de etanol e submeteu-se a mistura à agitação por 45 min, à temperatura ambiente. Em seguida, centrifugou-se a $3.000 \mathrm{rpm}$, durante $5 \mathrm{~min}$. O precipitado (polímero) foi descartado e o sobrenadante foi submetido à evaporação em evaporador rotatório, para retirada do solvente orgânico, obtendo-se um segundo resíduo.

A separação dos outros componentes tensoativos, óleo, diclofenaco e produtos de degradação, foi realizada em coluna cromatográfica preparativa, com gel de sílica (70-230 mesh), eluída sob pressão positiva com uma mistura de ciclohexano:acetato de etila na proporção de 4:1 (v/v). O segundo resíduo em balão de fundo redondo foi dissolvido em $5 \mathrm{~mL}$ do eluente, aplicados à coluna e eluídos com $200 \mathrm{~mL}$ da mistura eluente. Foram coletadas 30 frações, que foram submetidas à análise através de cromatografia em camada delgada (cromatofolhas de alumínio, gel de sílica $60 \mathrm{~F}_{254}$ ). O eluente para a cromatografia em camada delgada foi idêntico ao eluente utilizado na cromatografia em coluna, e os cromatogramas foram revelados com uma mistura de vanilina/metanol/água e aquecimento. As frações de composição semelhante foram reunidas e evaporadas até a secura, em evaporador rotatório.

Para purificação dos produtos de degradação, utilizou-se cromatografia em camada delgada preparativa $(5 \mathrm{~mm})$, com gel de sílica de granulometria superior a $0,02 \mathrm{~mm}$. As placas foram recortadas nas bandas correspondentes aos produtos de degradação. As bandas recortadas foram dispersas, separadamente, em acetonitrila e submeti- das à agitação em agitador magnético durante 30 min, na temperatura de $40{ }^{\circ} \mathrm{C}$. Em seguida, foram submetidas à filtração sobre gel de sílica (230-400 mesh). Os filtrados correspondentes foram evaporados à secura, sob pressão reduzida em evaporador rotatório.

\section{Identificação dos produtos de degradação isolados}

Dois produtos de degradação do diclofenaco foram isolados e analisados qualitativamente por CLAE (fase móvel acetonitrila:água 65:35 v/v), ajustada a $\mathrm{pH}$ aparente 5,0 com ácido acético $10 \%$, fluxo de $0,7 \mathrm{~mL} / \mathrm{min}$ e volume injetado de amostra de $20 \mu \mathrm{L}$ ) e cromatografia gasosa acoplada à espectrometria de massas em um equipamento Varian modelo SATURNO 2000. Para análise por cromatografia gasosa, as amostras foram dissolvidas em acetonitrila, e um volume de $1 \mu \mathrm{L}$ foi injetado no modo "splitless" na temperatura do injetor de $250{ }^{\circ} \mathrm{C}$, em uma coluna capilar DB5 (30 m $\times 0,25 \mathrm{~mm}$ $\times 0,25 \mu \mathrm{m}$ ) nas seguintes condições: $100{ }^{\circ} \mathrm{C}$ por $1 \mathrm{~min}$, seguido de taxa de aquecimento de $10 \% \mathrm{~min}$ até $280^{\circ} \mathrm{C}$.

\section{Análises estatísticas}

Os dados experimentais foram analisados estatisticamente através da análise de variância (ANOVA).

\section{RESULTADOS E DISCUSSÃO}

\section{Estabilidade do diclofenaco nas suspensões e nos pós frente ao armazenamento}

Com o objetivo de estabilizar as formulações de suspensões e pós de nanocápsulas contendo diclofenaco, o monoestearato de sorbitano foi substituído por Epikuron $170^{\circledR}$, cuja aplicabilidade na preparação de suspensões de nanocápsulas é descrita na literatura ${ }^{31,35}$. As Tabelas 2 e 3 descrevem a recuperação do diclofenaco, em função do tempo de armazenamento, a partir das suspensões e dos pós correspondentes, respectivamente.

Todas as suspensões apresentaram-se viáveis, com exceção da formulação sem ambos os tensoativos (Tabela 1, suspensão G), que apresentou aglomerados macroscópicos antes mesmo da evaporação do solvente orgânico. A formulação, contendo apenas o Tween $80^{\circledR}$ como tensoativo (suspensão F), apresentou simultaneamente nanopartículas inferiores a $400 \mathrm{~nm}$ e uma pequena quantidade de precipitado macroscópico após evaporação do solvente orgânico e concentração da água. A análise do tamanho de partícula das demais suspensões (A-E) demonstrou que todas se apresentaram nanométricas monomodais, com diâmetros inferiores a $300 \mathrm{~nm}$ (Tabela 2).

As suspensões A e B permaneceram estáveis quimicamente durante os 14 meses de estudo, apresentando recuperação do diclofenaco constante $(\alpha=0,05)$ (Tabela 2). Por outro lado, a suspensão C e a

Tabela 2. Diâmetro médio de partícula após preparação e recuperação do diclofenaco das suspensões de nanocápsulas, preparadas com concentrações decrescentes dos tensoativos, em função do tempo

\begin{tabular}{cccccc}
\hline Suspensão & Diâmetro $(\mathrm{nm})$ & \multicolumn{3}{c}{ Doseamento em função do tempo $(\mathrm{mg} / \mathrm{mL} \pm \mathrm{s})$} \\
& & 0 meses & 3 meses & 6 meses & 14 meses \\
\hline A & $182 \pm 28$ & $1,06 \pm 0,02$ & $1,02 \pm 0,04$ & $1,08 \pm 0,04$ & $1,03 \pm 0,02$ \\
B & $181 \pm 51$ & $1,11 \pm 0,09$ & $1,07 \pm 0,03$ & $1,02 \pm 0,02$ & $1,00 \pm 0,02$ \\
C & $186 \pm 51$ & $1,03 \pm 0,04$ & $1,01 \pm 0,02$ & $1,05 \pm 0,03$ & $0,94 \pm 0,03$ \\
D & $224 \pm 55$ & $1,00 \pm 0,01$ & $0,89 \pm 0,04 *$ & $0,94 \pm 0,08 *$ & $0,82 \pm 0,08 *$ \\
E & $179 \pm 49$ & $1,07 \pm 0,10$ & $1,02 \pm 0,05$ & $1,05 \pm 0,01$ & $0,90 \pm 0,05$ \\
F & $292 \pm 90$ & $0,96 \pm 0,01 *$ & $0,70 \pm 0,16 *$ & $0,61 \pm 0,10 *$ & $0,54 \pm 0,15 *$ \\
\hline
\end{tabular}

* Aparecimento de picos adicionais nos cromatogramas. 
Tabela 3. Recuperação do diclofenaco, em função do tempo, dos pós-obtidos a partir das suspensões de nanocápsulas preparadas com concentrações decrescentes dos tensoativos

\begin{tabular}{ccccc}
\hline Pós & \multicolumn{4}{c}{ Recuperação do diclofenaco $(\% \pm \mathrm{s}) * *$} \\
& 0 meses & 3 meses & 6 meses & 14 meses \\
\hline A & 100,0 & $96,8 \pm 3,9$ & $92,2 \pm 6,1$ & $91,7 \pm 0,1$ \\
B & 100,0 & $95,3 \pm 5,4$ & $91,3 \pm 5,7$ & $93,1 \pm 7,5$ \\
C & 100,0 & $100,5 \pm 0,6$ & $101,9 \pm 1,3$ & $96,1 \pm 2,9$ \\
D & 100,0 & $97,2 \pm 2,6$ & $96,1 \pm 3,6$ & $93,2 \pm 3,8$ \\
E & 100,0 & $100,0 \pm 8,1$ & $99,8 \pm 8,3$ & $97,5 \pm 3,6$ \\
F & 100,0 & $89,6 \pm 6,1^{*}$ & $72,7 \pm 12,8^{*}$ & $65,7 \pm 0,5^{*}$ \\
\hline
\end{tabular}

* Aparecimento de picos adicionais nos cromatogramas.

** Considerando-se percentual em relação à concentração no tempo zero.

suspensão E (sem o Tween $80^{\circledR}$ ) apresentaram um discreto declínio na taxa de recuperação do diclofenaco, principalmente entre o sexto e o décimo quarto meses, porém não foi observado pico adicional nos cromatogramas. Este fato provavelmente deve-se à precipitação simultânea de nanocristais durante a formação das nanocápsulas, estabilizados pelos tensoativos, quando a concentração do diclofenaco excede sua concentração de saturação para associação nos colóides. Segundo Guterres e colaboradores ${ }^{31}$, estes nanocristais apresentam a mesma distribuição de tamanho de partícula das nanocápsulas e, em função do tempo de armazenamento, eles se agregam e precipitam.

A suspensão D também apresentou declínio na taxa de recuperação do diclofenaco, acompanhado do aparecimento de picos adicionais nos cromatogramas a partir do terceiro mês, com tempos de retenção de 4,1 min e de 6,2 min, correspondentes àqueles observados anteriormente por Müller ${ }^{17}$. Desta forma, a diminuição do teor de fármaco não pode ser unicamente devida à formação simultânea de nanocristais durante a preparação da formulação mas, também, devida ao início da degradação do fármaco, concomitantemente. Este resultado também pode ser observado para a suspensão $\mathrm{F}$ (sem o Epikuron $\left.170^{\circledR}\right)$, logo após a preparação da formulação, observado pelo decréscimo significativo $(\alpha=0,05)$ de aproximadamente $50 \%$ na recuperação do diclofenaco até o décimo quarto mês, com aparecimento dos picos adicionais no cromatograma. Estes resultados podem indicar a degradação do diclofenaco nestas duas suspensões e que a presença do Epikuron $170^{\circledR}$ poderia exercer um efeito protetor do fármaco, estabilizando-o nas formulações.

A secagem de suspensões de nanopartículas normalmente é empregada visando-se o interesse de aplicação industrial dos produtos secos pela diminuição da contaminação microbiana, pela viabilidade tecnológica como intermediários de formas sólidas finais, assim como pelo aumento da estabilidade físico-química dos produtos ${ }^{36-39}$. As suspensões A-F foram submetidas à secagem por aspersão logo após preparação, utilizando-se o Aerosil $200^{\circledR}$ como adjuvante de secagem. Os rendimentos da operação situaram-se entre 50 e $58 \%$.

A recuperação do diclofenaco a partir dos pós A-E, em função do tempo, demonstrou que o seu teor mantém-se constante nos 14 meses do estudo de estabilidade $(\alpha=0,05)$ (Tabela 3 ). O pó $\mathrm{F}$ (sem Epikuron $\left.170^{\circledR}\right)$ apresentou um decréscimo significativo $(\alpha=0,05)$ na recuperação do diclofenaco a partir do terceiro mês observandose, simultaneamente, o aparecimento dos picos adicionais nos cromatogramas.

A análise dos resultados demonstra que a estabilidade dos colóides é afetada pelo tipo e concentração dos tensoativos, sendo que as suspensões mostraram-se mais instáveis que os seus produtos secos correspondentes. Também pode-se verificar, tanto para as formas líquidas como para as sólidas, que a presença de Epikuron $170^{\circledR}$ acima da concentração de $3,06 \mathrm{mg} / \mathrm{mL}$, na preparação das suspensões, impede a diminuição do teor de diclofenaco, seja evitando a agregação do fármaco e/ou exercendo um efeito protetor contra uma possível degradação do diclofenaco, uma vez que as formulações preparadas na sua ausência (suspensão e pó F) apresentaram diminuição significativa $(\alpha=0,05)$ do fármaco, com o aparecimento de picos adicionais nos cromatogramas.

\section{Experimentos de degradação}

Um estudo de fotodegradação foi realizado com lâmpada UVC, mantendo-se a temperatura interna da câmara em $28^{\circ} \mathrm{C} \pm 1^{\circ} \mathrm{C}$, para se investigar os possíveis fatores que conduzem à diminuição do teor do fármaco, acompanhada de aparecimento de picos adicionais nos cromatogramas (CLAE), anteriormente observados no estudo prévio de estabilidade de suspensões de nanocápsulas contendo diclofenaco.

Para verificar a influência da temperatura da câmara sobre a degradação do diclofenaco, as formulações foram também submetidas ao ensaio protegidas da luz com papel alumínio. Quando analisadas por CLAE, não apresentaram pico além do sinal relativo ao diclofenaco, o que sugere que a temperatura no interior da câmara não influenciou na degradação do fármaco.

Cada componente da formulação de nanocápsulas foi analisado por CLAE isoladamente. O Miglyol 810 ${ }^{\circledR}$, o Epikuron $170^{\circledR} \mathrm{e}$ a poli $(\varepsilon-$ caprolactona) não apresentaram absorção no comprimento de onda avaliado. Por sua vez, o cromatograma do Tween $80^{\circledR}$ apresentou um pico em 7,3 min. Quando expostas à luz UVC isoladamente, todas estas quatro matérias-primas das formulações de nanocápsulas não apresentaram picos detectáveis ou adicionais na análise por CLAE.

O diclofenaco ácido no estado sólido foi submetido à luz UVC, não tendo sido observados picos adicionais nos cromatogramas (CLAE, $280 \mathrm{~nm}$ ) até 14 dias de exposição à luz UVC.

Na Figura 1a, pode-se observar o cromatograma da suspensão $F$ (sem o Epikuron $170^{\circledR}$ ) após preparação. Antes da exposição à luz UVC, o cromatograma apresenta apenas o pico referente ao diclofe-

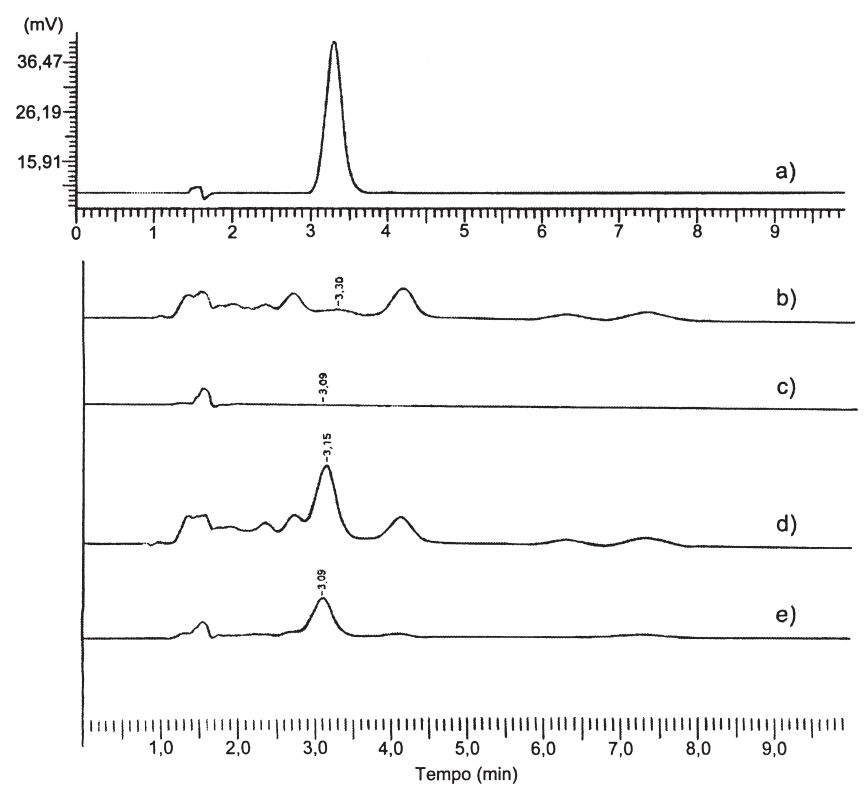

Figura 1. a) Cromatograma da suspensão de nanocápsulas $F$, formulada sem Epikuron $170^{\circledR}$, antes da exposição à luz UVC. b) e c) Cromatogramas obtidos após 14 dias de exposição à luz UVC, da suspensão de nanocápsulas $F$ e do produto seco $F$, respectivamente. d) e e) Cromatogramas obtidos após 14 dias de exposição à luz UVC, da suspensão de nanocápsulas A e do produto seco $A$, respectivamente 
naco, em 3,2 min. As demais formulações apresentaram cromatogramas semelhantes após preparação.

Com apenas 1 dia de exposição, para a suspensão $F$ foram observados no cromatograma picos adicionais com tempos de retenção em 4,1 min e em 6,2 min após análise de uma alíquota por CLAE, que aumentam de área com o tempo de exposição à luz UVC. Estes picos coincidem com aqueles observados anteriormente nas suspensões de nanocápsulas de diclofenaco em função do tempo. Além destes picos, pode-se observar, nas amostras submetidas à luz UVC, picos adicionais com tempos de retenção inferiores a 3,2 min, que não haviam sido observados no estudo prévio de estabilidade. Ainda pode-se observar um pico em 7,3 min, proveniente do Tween $80^{\circledR}$. Após 3 dias de exposição, verifica-se o desaparecimento do pico referente ao diclofenaco. As Figuras 1b e 1c mostram os cromatogramas das formulações $\mathrm{F}$ (suspensão e pó), preparadas sem Epikuron $170^{\circledR}$, após 14 dias de exposição à luz UVC.

Por outro lado, quando a suspensão A (Tabela 1) foi submetida à exposição à luz UVC durante 14 dias, foi observado o aparecimento dos picos com tempos de retenção em 4,1 min e em 6,2 min, observando-se também o pico relativo ao diclofenaco em 3,2 min (Figura 1d). Quando o produto seco A foi submetido à exposição à luz UVC (Figura 1e), também se pode observar a presença destes picos em 3,2 min, correspondendo ao diclofenaco, e em 4,1 min e 6,2 min. Estes resultados sugerem o efeito protetor dos fosfolipídeos presentes nas formulações A (Epikuron $170^{\circledR}$ ), denotado pela presença do diclofenaco nestas formulações após 14 dias de exposição à luz UVC, quando comparado às formulações F (suspensão e pó, sem Epikuron $170^{\circledR}$ ).

Para confirmar a potencialidade do Epikuron $170^{\circledR}$ na estabilização química do diclofenaco, misturas binárias de cada componente da formulação com o fármaco foram expostas à luz UVC. Quando a mistura binária diclofenaco ácido e Miglyol $810^{\circledR}$ foi submetida ao ensaio, foi verificado o aparecimento do pico em 4,1 min no cromatograma, logo após as primeiras 24 h de irradiação. Após 3 dias de exposição à luz UVC, além dos picos em 3,2 min e em 4,1 min, o pico em 6,2 min também foi observado. Este mesmo comportamento pode ser verificado para as misturas binárias diclofenaco ácido e poli( $\varepsilon$-caprolactona) e diclofenaco ácido e Tween $80^{\circledR}$. No entanto, quando a mistura binária diclofenaco ácido e Epikuron $170^{\circledR}$ foi submetida ao ensaio de exposição à luz UVC, não se verificou o aparecimento dos picos adicionais nos cromatogramas, nem mesmo após os 14 dias de exposição à luz UVC, mantendo-se constante a área do pico relativo ao diclofenaco. Este fato evidencia que o Epikuron $170^{\circledR}$ exerce um efeito protetor sobre o diclofenaco, impedindo e/ou retardando a sua degradação. Provavelmente este efeito protetor verificado para o Epikuron $170^{\circledR}$ deve-se à sua estrutura fosfolipídica, a qual, numa formulação de suspensões coloidais, pode conduzir à formação concomitante de nanocápsulas e de lipossomas. Mosqueira e colaboradores ${ }^{40}$ verificaram, para suspensões de nanocápsulas de ácido poli(D,L-láctico), nas quais o Epikuron $170^{\circledR}$ foi utilizado como tensoativo, a formação de lipossomas multilamelares e estruturas formadas de multicamadas, concomitante à formação das nanocápsulas. Dessa forma, o fármaco poderia ser protegido da luz pela presença de Epikuron $170^{\circledR}$ na formulação numa concentração acima de $3,06 \mathrm{mg} / \mathrm{mL}$ (Tabela 2), seja através da adsorção do fosfolipídeo às nanocápulas contendo diclofenaco, seja pela formação concomitante de lipossomas contendo o fármaco ou, ainda, pela formação de uma estrutura lipossômica englobando as nanocápsulas. Esta proteção estaria relacionada à composição química do Epikuron $170^{\circledR}$, composto de uma mistura de glico- e fosfolipídeos, que são ésteres de ácidos graxos saturados e insaturados, como o ácido linoleico. Desta forma, a reação de oxidação das cadeias insaturadas do Epikuron $170^{\circledast}$ ocorre preferencialmente à degradação do diclofenaco.

\section{Isolamento e identificação dos produtos de degradação do diclofenaco}

Um produto de nanocápsulas, preparadas com monoestearato de sorbitano e Tween $80^{\circledR}$ (sem Epikuron $170^{\circledR}$ ), seco por aspersão, contendo diclofenaco e armazenado durante 2 anos, após extração com acetonitrila e análise por CLAE, apresentou os mesmos picos com tempos de retenção em 4,1 min e em 6,2 min. Estes compostos [composto (I) e composto (II)] foram isolados desta amostra, conforme descrito na parte experimental, e analisados novamente por CLAE, apresentando os tempos de retenção de 4,1 min [composto isolado (I)] e de 6,2 min [composto isolado (II)]. Analisou-se também (CLAE) a co-injeção de cada composto isolado (I) ou (II) com uma amostra de suspensão de nanocápsulas, apresentando os picos de degradação, não se verificando sinais além daqueles presentes no cromatograma da suspensão, havendo completa sobreposição de cada pico com tempo de retenção em 4,1 min ou 6,2 min para cada análise. Dessa forma, pode-se concluir que os compostos isolados (I) e (II) são produtos idênticos aos detectados na análise de amostras de suspensões $\mathrm{D}$ e $\mathrm{F}$ e do pó $\mathrm{F}$, contendo diclofenaco, armazenados por mais de três meses.

Com a finalidade de identificar estes dois compostos isolados, os mesmos foram submetidos à análise por cromatografia gasosa, acoplada à espectrometria de massas. Estes apresentaram tempos de retenção em, respectivamente, 12,9 $\min$ [composto isolado (I)] e 12,3 min [composto isolado (II)]. Os espectros de massas (Figura 2) foram analisados, e o composto (I) apresentou o íon molecular $[\mathrm{M}]^{+\bullet}$ a $267 \mathrm{~m} / \mathrm{z}$ com abundância relativa de $25 \%$, assim como o íon $[\mathrm{M}+2]^{+\bullet}$ com abundância relativa de $17 \%$ e o íon $[\mathrm{M}+4]^{+\bullet} \operatorname{com} 3 \%$, indicando a presença de dois átomos de cloro na molécula. $\mathrm{O}$ pico base com $214 \mathrm{~m} / \mathrm{z}$ é decorrente das perdas de $\mathrm{HCl}$ e de $\mathrm{OH}$, e a presença do pico em $216 \mathrm{~m} / \mathrm{z}$ (36\%) indica um átomo de cloro no fragmento. O pico em $179 \mathrm{~m} / \mathrm{z}(17 \%)$ corresponde a um fragmento com perda de dois átomos de cloro e 18 u.m.a. a partir do íon molecular. A análise sugere que o composto (I) corresponde ao álcool 2-(2',6'-diclorofenil)aminobenzílico (Figura 3), produto de degradação do diclofenaco, que já havia sido relatado por Sun e Fabre $^{30}$ e verificado por Cwiertnia e colaboradores ${ }^{25}$, para amostras de diclofenaco submetidas à temperatura de $80^{\circ} \mathrm{C}$.

O composto (II) apresentou o íon molecular [M] ${ }^{+}$a $265 \mathrm{~m} / \mathrm{z}$ com abundância relativa de $64 \%$, assim como o íon $[\mathrm{M}+2]^{+\bullet}$ com abundância relativa de $40 \%$ e o íon $[\mathrm{M}+4]^{+\bullet}$ com $5 \%$, indicando a presença de dois átomos de cloro na molécula. $\mathrm{O}$ pico base com $230 \mathrm{~m} / \mathrm{z}$ é decorrente da perda de um átomo de cloro e o pico em
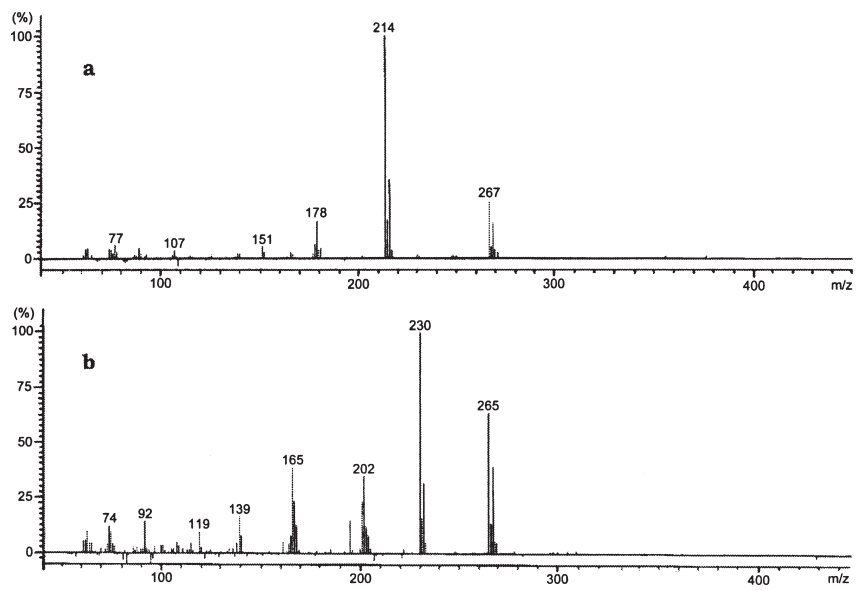

Figura 2. Espectros de massas do composto isolado (I) (a) e do composto isolado (II) (b) 
<smiles>O=C(O)Cc1ccccc1Nc1c(Cl)cccc1Cl</smiles>

Diclofenaco

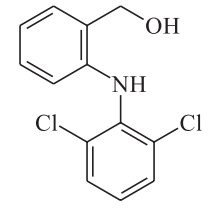

(I)

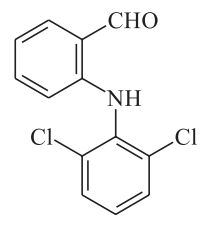

(II)
Figura 3. Estruturas químicas do diclofenaco e dos compostos de degradação do diclofenaco, (I) álcool 2-(2',6'-diclorofenil)aminobenzílico $e($ II) N-(2',6'-diclorofenil)antranilaldeído

$202 \mathrm{~m} / \mathrm{z}(36 \%)$ corresponde à perda de CO. O pico em 166 m/z (40\%) corresponde a um fragmento com perda de dois átomos de cloro e 29 u.m.a. a partir do íon molecular. A análise sugere que o composto (II) corresponde ao $\mathrm{N}$-(2',6'-diclorofenil)antranilaldeído (Figura 3), produto que também já foi descrito por alguns autores como um dos produtos de degradação do diclofenaco sódico, formado por uma descarboxilação oxidativa do fármaco ${ }^{25,26,41}$.

\section{CONCLUSÕES}

O diclofenaco formulado em suspensões de nanocápsulas e seus produtos secos por aspersão sofrem diminuição do teor em função do tempo de armazenamento. Esta redução é dependente do tipo e da concentração do sistema tensoativo, utilizado na preparação das formulações. A presença de Epikuron $170^{\circledR}$ em concentração superior a $3,06 \mathrm{mg} / \mathrm{mL}$ estabiliza física- e quimicamente o fármaco, previne sua diminuição em teor e o aparecimento de produtos de degradação nas formulações.

Estudos de degradação acelerada do diclofenaco nas suspensões e pós-correspondentes, através de exposição à luz UVC dessas formulações e de misturas binárias de cada componente com o fármaco, confirmaram o efeito de estabilização química do diclofenaco promovido pelo Epikuron $170^{\circledR}$.

A análise através de cromatografia gasosa acoplada à espectrometria de massas permitiu identificar os produtos de degradação do diclofenaco, nas formulações de nanocápsulas poliméricas formuladas com baixa concentração (menor que $3,06 \mathrm{mg} / \mathrm{mL}$ ) ou ausência de Epikuron $170^{\circledR}$, como sendo o álcool 2-(2',6'-diclorofenil)aminobenzílico e o $\mathrm{N}$-(2',6'-diclorofenil)antranilaldeído.

\section{AGRADECIMENTOS}

\section{À FAPERGS, ao CNPq/MCT e à Rede Nanobiotec/CNPq.}

\section{REFERÊNCIAS}

1. O'Brien, W. M.; Am. J. Med. 1986, 80, suppl 4B, 70.

2. Gennaro, A. R., ed.; Remington: The Science and Practice of Pharmacy, $19^{\mathrm{a}}$ ed.; Easton: Mack, vol. 2, 1995.

3. Lehtola, J.; Sipponen, P.; Scand. J. Rheumat. 1977, 6, 97.
4. Uthgenannt, H.; Letzel, H.; Br. J. Clin. Pract. 1981, 35, 229.

5. Kobyashi, K.; Arakawa, T.; Satoh, H.; Fukuda, T.; Nakamura, H.; Prostaglandins 1985, 30, 609.

6. Todd, P. A.; Sorkin, E. M.; Drugs 1988, 35, 244

7. Fara, J. W.; Myrback, R. E.; Pharm. Res. 1990, 7, 616.

8. Cioli, V.; Putzolu, S.; Rossi, V.; Scorza Barcellona, P.; Corradino, C.; Toxicol. Appl. Pharmacol. 1979, 50, 283.

9. Liversidge, G. G.; Conzentino, P.; Int. J. Pharm. 1995, 125, 309.

10. Story, M. J.; Flynn, M. J.; Eur. pat. 0274 870/A2, 1987.

11. Weiner, A. L.; Cullis, P. R.; Eur. pat. 0249 561/A2, 1987.

12. Franz, M.; Jans, M.; Mathur, L. K.; Shah, K. B. Truelove, J. E.; US pat. 5,015,481, 1991.

13. Nekroshus, E. S.; Rerhetnyak, V. Y.; Farmatsiya 1989, 38, 29.

14. Hasan, M.; Najib, N.; Suleiman, M.; El-Sayed, Y.; Abdel-Hamid, M.; Drug Dev. Ind. Pharm. 1992, 18, 1981.

15. Yang-Lin, S.; Horng-Kao, Y.; Pharm. Res. 1991, 8, 919.

16. Guterres, S. S.; Fessi, H.; Barratt, G.; Puisieux, F.; Devissaguet, J-P.; Pharm. Res. 1995, 12, 1.

17. Müller, C. R.; Dissertação de Mestrado, Universidade Federal do Rio Grande do Sul, Brasil, 1999.

18. Guterres, S. S.; Müller, C. R.; Michalowski, C. B.; Pohlmann, A. R.; Dalla Costa, T.; S.T.P. Pharma Sci. 2001, 11, 229.

19. Guterres, S. S.; Pohlmann, A. R.; Dalla-Costa, T.; Bassanni, V. L.; Müller, C. R.; Br PI 9906081-7, 1999.

20. Schaffazick, S. R.; Dissertação de Mestrado, Universidade Federal do Rio Grande do Sul, Brasil, 2001.

21. Kubala, T.; Gambhir, B.; Borst, S. I.; Drug Dev. Ind. Pharm. 1993, 19, 749.

22. Karamancheva, I.; Dobrev, I.; Brakalov, L.; Andreeva, A.; Anal. Lett. 1997, 30,2235 .

23. Karamancheva, I.; Dobrev, I.; Brakalov, L.; Andreeva, A.; Anal. Lett. 1998, $31,117$.

24. Buser, H. R.; Poiger, T.; Müller, M. D.; Environ. Sci. Technol. 1998, 32, 3449 .

25. Cwiertnia, B.; Hladon, T.; Stobiechi, M.; J. Pharm. Pharmacol. 1999, 51, 1213.

26. Hladon, T.; Cwiertnia, B.; Pharmazie 1999, 54, 943.

27. Eyjolfsson, R.; Drug Dev. Ind. Pharm. 2000, 26, 451.

28. Zwiener, C.; Frimmel, F. H.; Water Res. 2000, 34, 1881

29. Poiger, T.; Buser, H. R.; Müller, M. D.; Environ. Toxicol. Chem. 2001, 20, 256.

30. Sun, S. W.; Fabre, H.; J. Liq. Chromatogr. Relat. Technol. 1994, 17, 433.

31. Guterres, S. S.; Fessi, H.; Barratt, G.; Devissaguet, J-P.; Puisieux, F.; Int. J. Pharm. 1995, 113, 57.

32. Fessi, H.; Puisieux, F.; Devissaguet, J-P.; Eur. pat. 0274961 Al, 1988.

33. Müller, C. R.; Bassani, V. L.; Pohlmann, A. R.; Michalowski, C. B.; Petrovick, P. R.; Guterres, S. S.; Drug Dev. Ind. Pharm. 2000, 26, 343.

34. Fallavena, P. R. B.; Tese de Doutorado, Universidade Federal do Rio Grande do Sul, Brasil, 1998.

35. Ammoury, N.; Fessi, H.; Devissaguet, J-P.; Allix, M.; Plotkine, M.; Boulu, R. G.; J. Pharm. Pharmacol. 1990, 42, 558.

36. Chasteigner, S.; Fessi, H.; Cavé, G.; Devissaguet, J-P.; Puisieux, F.; S.T.P. Pharma Sci. 1995, 5, 242.

37. Quintanar-Guerrero, D.; Allémann, E.; Fessi, H.; Doelker, E.; Pharm. Res. 1998, 15, 1056.

38. Schaffazick, S. R.; Pohlmann, A. R.; Freitas, L. L.; Guterres, S. S.; Acta Farm. Bonaerense 2002, 21, 99

39. Pohlmann, A. R.; Weiss, V.; Mertins, O.; Pesce da Silveira, N.; Guterres, S. S.; Eur. J. Pharm. Sci. 2002, 16, 305.

40. Mosqueira, V. C. F.; Legrand, P.; Pinto-Alphandary, H.; Puisieux, F.; Barratt, G.; J. Pharm. Sci. 2000, 89, 614

41. Lewis, L.; Boni, R. L.; Adeyeye, C. M.; J. Microencapsulation 1998, 15, 555 . 